\title{
Technical Standards and Guidelines for Fragile X: The First of a Series of Disease-Specific Supplements to the Standards and Guidelines for Clinical Genetics Laboratories of the American College of Medical Genetics
}

Anne Maddalena, PhD ${ }^{\star 1}$, Carolyn Sue Richards, PhD, Co-Chair ${ }^{\star 2}$, Matthew J. McGinniss, PhD ${ }^{\star 3}$, Arthur Brothman, PhD, Co-Chair ${ }^{4}$, Robert J. Desnick, $M D, P h D^{5}$, Robert E. Grier, PhD ${ }^{6}$, Betsy Hirsch, $P h D^{7}$, Peter Jacky, PhD ${ }^{8}$, Geraldine A. McDowell, PhD ${ }^{9}$, Bradley Popovich, $P h D^{10}$, Michael Watson, $P h D^{11}$, and Daynna J. Wolff, PhD ${ }^{12}$ (Quality Assurance Subcommittee of the Laboratory Practice Committee)

Preface: The Quality Assurance subcommittee of the ACMG Laboratory Practice committee has the mission of maintaining high technical standards for the performance and interpretation of genetic tests. In part, this is accomplished by the publication of the document "Standards and Guidelines for Clinical Genetics Laboratories," which was published in its second edition in 1999 and is now maintained online (see www.faseb.org/genetics/acmg/ index.html). This subcommittee also reviews the outcome of national proficiency testing in the genetics area and may choose to focus on specific diseases or methodologies in response to those results. Accordingly, the subcommittee selected fragile X syndrome to be the first topic in a new series of supplemental sections, recognizing that it is one of the most frequently ordered genetic tests and that it has many alternative methods with different strengths and weaknesses. This document follows the outline format of the general Standards and Guidelines. It is designed to be a checklist for genetic testing professionals who are already familiar with the disease and the methods of analysis.

\section{INTRODUCTION}

Disease-specific statements are intended to augment the current general ACMG Standards and Guidelines for Clinical Genetics Laboratories. Individual laboratories are responsible for meeting the CLIA/CAP quality assurance standards with respect to appropriate sample documentation, assay validation, general proficiency, and quality control measures.

\footnotetext{
From the ${ }^{1}$ Genetics \& IVF Institute, ${ }^{2}$ Baylor College of Medicine, ${ }^{3}$ SEQUENOM, Inc., ${ }^{4}$ University of Utah School of Medicine, ${ }^{5}$ Mount Sinai School of Medicine, ${ }^{6}$ Baylor University Medical Center in Dallas, ${ }^{7}$ University of Minnesota Medical School, ${ }^{8}$ Kaiser Permanente, NW, Portland, ${ }^{9}$ LabCorp, ${ }^{10}$ Oregon Health Sciences University, ${ }^{11}$ Washington University School of Medicine, and ${ }^{12}$ Medical University of South Carolina.

${ }^{*}$ Fragile X Molecular Working Group.

Ad hoc contributor: Karen Snow, PhD, Mayo Clinic (also with the Fragile X Molecular Working Group).
}

\section{BACKGROUND ON FRAGILE X SYNDROME}

2.1 Gene symbol/chromosome locus: FMR1; also FRAXA. 2.2 OMIM number: 309550.

2.3 Brief clinical description: The features of fragile $\mathrm{X}$ syndrome include mental retardation and certain characteristic but nonspecific physical features and behaviors. For more information see the online Gene Clinics profile at www.geneclinics.org and the National Fragile $\mathrm{X}$ Foundation at www.nfxf.org.

2.4 Mode of inheritance: Inheritance is X-linked but is also notable for the presence of unaffected male carriers. In typical fragile $\mathrm{X}$ families, the mutation is a multi-step expansion in a region of CGG repeats. Small expansions (premutations) are generally silent in both males and females. Large expansions (full mutations) are penetrant in all males and many females. With extremely rare exceptions, only females can have affected children.

2.5 Gene description/normal gene product: The gene product is FMRP, fragile $\mathrm{X}$ mental retardation protein, a widely expressed RNA-binding protein of unknown function found at highest levels in the brain and testes.

2.6 Mutational mechanism/abnormal gene product: Fragile $\mathrm{X}$ syndrome is caused by the deficiency or absence of FMRP. Theoretically, this can occur through any type of deletion or inactivating mutation, but in well over $99 \%$ of cases there is an expansion of a segment of CGG repeats in the 5' UT region of FMR1. Large CGG expansions in this region are associated with hypermethylation and inhibition of transcription.

2.7 Listing of mutations: Mutations at locations other than the CGG repeat have been described. A listing can be found in the Human Gene Mutation Database at www.uwcm.ac.uk/ $u w \mathrm{~cm} / \mathrm{mg} / \mathrm{hgmd0}$.html. Guidelines for detecting these relatively rare mutations are beyond the scope of this document.

2.8 Ethnic association of common mutations: All major ethnic groups appear to be susceptible to expansion of the FMR1 CGG region. 


\subsection{Special testing considerations}

2.9.1 Sensitivity and specificity: CGG-repeat expansion mutations account for $>99 \%$ of cases of fragile X syndrome. Therefore, tests that effectively detect and measure the CGGrepeat region of the FMR1 gene are $>99 \%$ sensitive. Positive results are $100 \%$ specific, although a gray zone of CGG repeat sizes has been defined where the significance of carrier test results is inconclusive (see section 3.1.2). There are no known forms of FMRP deficiency that do not map to the FMR1 gene. Fragile X syndrome should not be confused with the unrelated mental retardation syndrome associated with the FRAXE locus.

2.9.2 Diagnostic versus predictive testing: This test is used for confirmative diagnosis and carrier detection. Positive results are considered diagnostic rather than predictive, inasmuch as penetrance is virtually $100 \%$ in males and the age of onset is not variable.

2.9.3 Prenatal testing: This test can be used for prenatal diagnosis in both amniotic fluid cells and chorionic villus samples (CVS). Laboratories offering CVS testing must be aware of this tissue's unique properties. (a) Methylation associated with lyonization is usually not present, and methylation associated with full mutations may or may not be present. In the past, the hypomethylated status of this locus in this tissue had been thought of as a limitation or possible source of confusion. To the contrary, because it is unwarranted to use methylation status or X-inactivation for phenotypic prediction of a full mutation, the possible hypomethylation of this tissue is no disadvantage, provided that the tissue-specific basis of the hypomethylation is understood. It is an acceptable option to omit methylation analysis entirely when testing CVS specimens. In the minor fraction of CVS cases with a result that is ambiguous between a large premutation and a small full mutation by size criteria alone, a follow-up amniocentesis may be required. (b) The degree of somatic variation in a full mutation "smear" has a wider range of possibilities than is typically seen in blood specimens, from very limited to extraordinarily diffuse. (c) Mosaicism between trophoblasts and somatic cells is theoretically possible. For this reason, when CVS results indicate a premutation, follow-up amniocentesis has been suggested to rule out mosaicism for a full mutation; however, there is no known occurrence of this type of mosaicism.

\section{GUIDELINES}

\subsection{Definition of normal and mutation categories}

3.1.1 Normal alleles have a range of $\sim 5$ to $\sim 44$. The most common length by far is 29 or 30 CGG repeats. Normal alleles have no meiotic or mitotic instability.

3.1.1.1 In stable normal alleles, the CGG region is interrupted by an AGG triplet after every 9 or 10 CGG repeats. The AGG triplets are thought to anchor the region during replication and prevent strand slippage. Direct testing for the AGG triplets is not routinely performed.

3.1.2 The range from $\sim 45$ to $\sim 54$ is a Gray Zone (Intermediate, Inconclusive, Borderline). Alleles in this range can be considered normal in the sense that women with alleles of this size have not been observed to have affected children. On the other hand, alleles of this size may be associated with fragile $\mathrm{X}$ syndrome in future generations or in distant relatives. Minor increases and decreases in repeat number can occur when alleles of this size are passed on, but there is no measurable risk of an affected child in the next generation. Alleles in this range can be referred to as premutations if they are confirmed by family studies to be traceable to a known full mutation or unambiguous premutation.

3.1.3 Premutation alleles range from $\sim 55$ to $\sim 200$ repeats. They show no somatic variation. They are not hypermethylated and are not associated with mental retardation. Women with alleles in this range are considered to be at risk for having affected children, although to date all known mothers of affected children have alleles of 59 repeats or higher.

3.1.3.1 The upper limit of premutations is sometimes said to be $\sim 230$. In fact, both numbers (200 and 230) are rough estimates derived from Southern blots where large premutations were measured with increases of 0.5 to $0.6 \mathrm{~kb}$, implying roughly 170 to 200 more triplet repeats than normal.

3.1.4 Full mutations have over 230 repeats, typically several hundred to several thousand repeats. There is usually broad somatic variation within each patient. Hypermethylation is typically present on most or all copies. The appearance of full mutations may vary in CVS, compared with blood and amniocytes: methylation may or may not be present, and the degree of somatic heterogeneity ranges from distinctly limited to extraordinarily diffuse.

3.1.5 Mosaicism due to de novo somatic mutations does not occur at the FMR1 CGG-repeat region, but size mosaics and methylation mosaics have been observed. When mosaicism is present, tissue-specific differences can be seen.

3.1.5.1 Size mosaics: This term refers to subpopulations of full mutations and premutations. Occasionally there also may be minor subpopulations with near normal or subnormal length.

3.1.5.2 Methylation mosaics: This term refers to full mutations with subpopulations that remain unmethylated.

\subsection{METHODOLOGICAL CONSIDERATIONS}

All general guidelines for Southern blots and polymerase chain reaction (PCR) in the ACMG Standards and Guidelines apply. The following additional details are specific for fragile X. For this test, there are many valid methods with different strengths and weaknesses. Laboratories very often need to use more than one method because no single method can detect all types of mutations equally well or with equal precision. When a given method is recognized to have certain weaknesses, it is assumed that a complementary method will also be used.

\subsubsection{Southern blots}

\subsubsection{Probe and restriction site combinations}

Several single- and double-enzyme options are commonly used, and several probes are available (Table 1). ${ }^{1-4}$ Other re- 
Table 1

Probe and restriction site combinations

\begin{tabular}{lll}
\hline $\begin{array}{l}\text { Primary restriction sites } \\
\text { and normal length }\end{array}$ & $\begin{array}{c}\text { Optional internal } \\
\text { methyl-sensitive sites }\end{array}$ & Probes and references \\
\hline $\begin{array}{l}\text { EcoRI, } 5.2 \mathrm{~kb} \\
\text { HindIII, 5.4 kb }\end{array}$ & EagI, BssHI, NruI, etc. & $\begin{array}{l}\text { StB12.3 (1), E5.1 (2), } \\
\text { OxIII, } 12 \mathrm{~kb}\end{array}$ \\
$\begin{array}{l}\text { PstI, } 1.0 \mathrm{~kb} \\
\mathrm{n}\end{array}$ & pfxa3 (4), Ox0.55 (3) \\
\hline
\end{tabular}

striction enzymes and probes can be used, if equivalence is demonstrated.

3.2.1.2 In general, small premutations are more easily detected when the normal fragment is small and/or electrophoretic migration is long, whereas large/diffuse full mutations are more easily detected when the normal fragment is large and/or electrophoretic migration is short.

3.2.1.3 Controls should be included to confirm the proper choice and activity of restriction enzymes and probe, and ideally should represent the more difficult-to-recognize genotypes. To verify digestion and hybridization parameters, a normal control will suffice. However, in fragile $\mathrm{X}$ blots the abnormal controls are extremely important because they provide quality control on the resolution of small premutations and the detectability of diffuse smears.

3.2.1.4 For female patients, it should be noted that the degree of separation between two differently sized normal alleles could appear identical with that between a normal and a premutation allele (e.g., 20 and 44 repeats vs. 35 and 59 repeats). A Southern blot with superior resolution and appropriate size standards or controls is required to distinguish between these possibilities. Alternatively, most PCR-based methods can provide the required resolution. Similar considerations apply to detection of premutation alleles in normal transmitting males.

3.2.1.5 Because full mutations can be extremely diffuse and faint, signal:noise ratios must be very good. Laboratories are advised to be aware of the many different appearances of full mutations. Full mutations are not likely to be overlooked in males, inasmuch as the normal signal will be absent (or light, in size mosaics), but full mutations can be easily missed in females if the background is poor.

3.2.1.6 Migration distances should be interpreted using a standard ladder such as lambda HindIII fragments or a set of carefully chosen, independently tested human references.

3.2.1.7 The following guidelines refer to methylation analysis using double digestion.

3.2.1.7.1 In tissues other than CVS, methylation analysis reveals the degree of hypermethylation in full mutations and shows the distribution of X-inactivation in any female with two distinguishable alleles. Neither type of methylation information is necessary for the interpretation of results, but detection of methylation can help discriminate between premutations and full mutations for the rare alleles that fall near the boundary, i.e., around 200 repeats.

3.2.1.7.2 In tissues other than CVS, methylation analysis confirms the chromosomal gender of patients, which may be useful for quality assurance. It also detects $46, \mathrm{X}$ and $47, \mathrm{XXY}$ subjects indirectly.

3.2.1.7.3 In tissues other than CVS, methylation analysis increases the difficulty of detecting females with small premutations who have highly skewed X-inactivation. Double digestion with a methyl-sensitive enzyme causes the signal from each allele in a female to be split into active and inactive bands, forming four bands in a carrier female. When $\mathrm{X}$-inactivation is balanced in a carrier the two active bands are readily seen, although the two inactive bands may comigrate. However, if $\mathrm{X}$-inactivation is heavily skewed, there will be only two visible bands. This is particularly challenging when the premutation is predominantly inactive, because then it appears only in the upper region of the gel where resolution is considerably poorer. For an example of a carrier with extremely skewed $\mathrm{X}$-inactivation, see Figure 1, lane 13. Lanes 3 and 4 show two females with oppositely skewed X-inactivation.

3.2.1.7.4 In CVS tissue, the FMR1 region usually does not have methylation associated with X-inactivation, and it may or may not have hypermethylation associated with full mutations. When testing CVS tissue, methylation analysis is optional. If done, it should never be used to predict the severity of a full mutation or the influence of $\mathrm{X}$-inactivation.

Incidentally, methylation analysis can serendipitously alert a laboratory to maternal cell contamination in chorionic villus

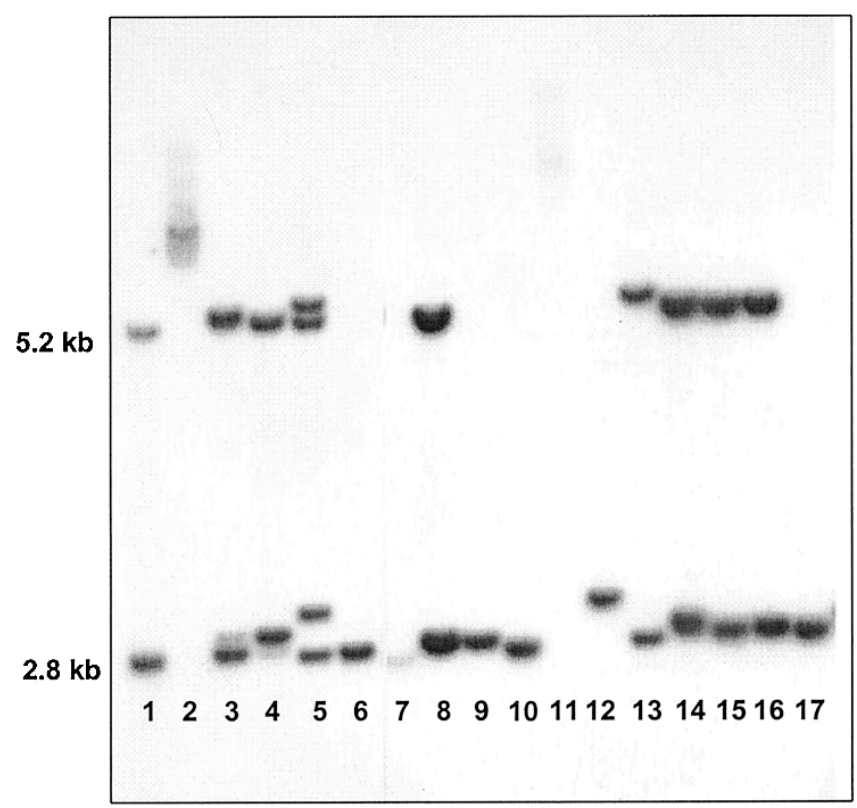

Fig. 1 Southern blot using EcoRI and EagI digestion, probed with StB12.3, using extended electrophoresis to illustrate several subtle specimen types. (1) Normal female. (2) Full mutation male. Note the combination of a predominant band with a diffuse smear. (3) Female with 28 and 52 repeats, with the smaller allele predominantly active. (4) Female with 26 and 52 repeats, with the larger allele predominantly active. (5) Female with 18 and $\sim 80$ repeats, with equal X-inactivation. (6) Normal male. (7) Normal male, underloaded and smiling due to DNA degradation. (The apparent line between lane 6 and 7 is a photographic artifact.) (8) Normal female. (9) Normal male. (10) Normal male. (11) Affected male, underloaded and very diffuse. (12) Premutation male. (13) Female with 20 and 70 repeats, with the smaller allele virtually exclusively active. The only evidence of abnormality is the slow migration of the " $5.2 \mathrm{~kb}$ " band. (14) Female with 27 and 42 repeats, with the larger allele somewhat more active. (15-17) Unremarkable normal females and male. Figure provided by Genetics \& IVF Institute. 
specimens: inasmuch as methylation associated with X-inactivation is usually not present at this locus in CVS tissue, a strong normal inactive band can be a sign of possible maternal cell contamination. Other explanations for such a band include $\mathrm{X}$-inactivation in some fetal cells or incomplete digestion. Further investigation would be called for.

\subsubsection{PCR methods}

3.2.2.1 Several sets of primers, PCR conditions, and methods of separation and detection have been published..$^{5-8}$ Other primers and methods can be used if equivalence is demonstrated. A particular region to be aware of in primer design is the deletional hotspot.

3.2.2.2 All PCR reactions, for any locus, can theoretically fail to detect an allele if there is polymorphism at a primer binding site. There are no known polymorphisms that would affect any of the commonly used primers.

3.2.2.3 Patient amplicon sizes should be determined using a standard ladder such as an M13 sequencing reaction or a set of carefully chosen, independently tested human references.

3.2.2.4 Controls representing the genotypes to be distinguished should be run on each gel. The upper limit of allele size that can be successfully detected should be known, and a control corresponding to that size should be included in each run. Laboratories should confirm the size of their control DNA by sequencing or by exchange with another laboratory.

3.2.2.5 Amplification of CG-rich regions is difficult, and special conditions are required. The difficulty increases with increasing numbers of CGG repeats; therefore, many PCR strategies do not attempt to detect large alleles. In such a system, it is not possible to tell the difference between a female who is homozygous for a normal allele and one who has a large nonamplifiable second allele. Similarly, patients who are mosaics for premutations and full mutations will appear to have only premutations.

3.2.2.6 When a PCR strategy is capable of detecting large alleles, amplification nevertheless may favor the smaller allele in any specimen with multiple alleles, i.e., females and mosaics. Such methods should be validated with carrier females and mosaics, in addition to males. Because of disproportionate amplification, PCR is not reliable for determining the ratio of different species in a mosaic individual.

3.2.2.7 In PCR amplification of females and mosaics, heteroduplexes can form. If denaturing electrophoresis is used, conditions must be sufficiently denaturing to avoid heteroduplex artifact. If nondenaturing electrophoresis is used, steps must be taken to distinguish between heteroduplexes and true abnormal alleles.

3.2.2.8 Basic PCR amplification is not affected by methylation. Although PCR tests specifically modified to detect methylation status have been described,, 910 the common PCR strategies that have been in use for many years are completely independent of methylation.

3.2.2.9 When a PCR strategy is used to detect full mutations, the presence of a deletion hotspot in the CGG-repeat region should be noted. ${ }^{11}$ Primers located within the deletion hotspot may result in failure to detect the expanded allele. Primers located upstream of the deletion hotspot may result in apparent size mosaicism.

\subsection{INTERPRETATIONS}

3.3.1 The following elements must be included in the report, in addition to the items described in the current general Standards and Guidelines.

3.3.1.1 State whether the method used was PCR, Southern blot, or both. If Southern blot, state the restriction enzymes and probes used. If PCR, state the method used for separation and detection.

3.3.1.2 State the definitions used for normal, gray-zone (borderline, inconclusive) premutation, and full mutation.

3.3.1.2.1 Note that it is not necessarily obvious that the borderline category refers to the border between normal and premutation, and not to the border between premutation and full mutation. Similarly, note that the term instability, which is often used with regard to borderline alleles to describe minor intergenerational or mitotic changes, may unintentionally suggest a risk of having an affected child or personal late-onset symptoms.

3.3.1.3 Classify the patient's result using the defined categories. The term size mosaic should be used for alleles that have significant subpopulations in both the premutation and full mutation range.

3.3.1.4 All positive results should state that genetic counseling is indicated and testing is appropriate for at-risk family members.

3.3.2 The following descriptive elements may appear, with caution.

3.3.2.1 The size of the alleles may be reported. If so, the precision used in quoting the size must be supportable by the precision of the ladder used, the sharpness of the bands or peaks, degree of stutter, etc. It may be appropriate to state a range or use qualifying terms such as approximately. Descriptions such as "positive for an allele with 55-200 repeats" are ambiguous.

3.3.2.2 Description of methylation may be provided. The two kinds of methylation must be clearly distinguished: methylation due to X-inactivation and hypermethylation of full mutations. The term methylation mosaic or incomplete methylation may be used if not all molecules in a full mutation are hypermethylated.

3.3.3 The following helpful points on alternative diagnoses may be included.

3.3.3.1 There are rare forms of FMRP deficiency not caused by CGG expansion, which may not be detected by this test.

3.3.3.2 Mental retardation associated with other fragile sites, in particular FRAXE, will not be detected with this test.

3.3.3.3 Routine chromosome analysis is recommended in the diagnostic workup of mental retardation.

3.3.4 Comments on phenotype, if included, should be abstract rather than case-specific. The following concepts apply. 
3.3.4.1 All males with full mutations have fragile X syndrome to some degree. The severity cannot be predicted from the size of the full mutation, but if premutations are also present or if the majority of the full mutation molecules are unmethylated, the phenotype MAY be less severe.

3.3.4.2 Females with full mutations have a wide spectrum of phenotypes ranging from as severely affected as a male (which is itself a range) to very mild or undetectable. The severity cannot be predicted from the size of the full mutation, nor can it be predicted from the pattern of X-inactivation.

3.3.4.3 Individuals with premutations should be interpreted as unaffected carriers. The only clearly established effect associated with premutations is a tendency in some women for premature ovarian failure. ${ }^{12}$ If a premutation is found in a developmentally delayed patient, it must be considered a coincidence unless FMRP deficiency or mosaicism for a full mutation can be found.

3.3.4.4 Individuals with gray-zone alleles should be interpreted as unaffected. Even more so than a premutation, a grayzone allele is considered a coincidence when found in a developmentally delayed patient. FMRP deficiency or mosaicism for a full mutation can be investigated, but with less likelihood of success because gray-zone alleles are not uncommon in the general population.

3.3.5 Comments on reproductive risk, if included, should be abstract rather than case-specific. The following concepts apply.

3.3.5.1 All affected males and the overwhelming majority of affected females inherit their mutations from their mothers. The mothers may have premutations or full mutations.

3.3.5.2 Women with full mutations have a theoretical 50\% chance of passing on the full mutation with each pregnancy.

3.3.5.3 Women with premutations have a theoretical 50\% chance of passing on the abnormal $\mathrm{X}$ with each pregnancy. If it is passed on, the chance the allele will increase to a full mutation depends on its size in the mother. Probabilities range from "too rare to measure" for maternal alleles in the mid 50s to nearly $100 \%$ for maternal alleles of 90 and above. The absolute allele size at which risk begins, and what that minimal risk is, are not clearly known. Laboratories should be familiar with the data of Fu et al. ${ }^{5}$ and Nolin et al. ${ }^{13}$ and any current publications on this topic.

3.3.5.4 Men with premutations will almost always pass premutations to all of their daughters. An extremely rare phenomenon involves unaffected males with premutations who have had affected daughters, apparently by gonadal mosaicism for full mutations. The sons of men with premutations are not at risk.

3.3.5.5 Men and women alike with gray-zone alleles are not known to have affected children in the next generation. Their status as carriers depends in part on whether the allele can be traced through the family to a known full mutation or unambiguous premutation. In the absence of such a connection, on a research basis it may be possible to show mitotic instability and/or loss of the AGG interruptions, but such data have little clinical value for predicting the risk to specific future generations.

\section{ALTERNATIVE TESTING METHODS}

4.1 Cytogenetic analysis: Testing for the fragile site FRAXA at $\mathrm{Xq} 27$ is no longer an acceptable diagnostic method. Specificity and sensitivity are both insufficient.

4.2 Protein analysis: Immunohistochemical staining for FMRP is a valid diagnostic method. As a carrier test it is limited to detecting full mutation carriers.

\section{POLICY STATEMENTS}

5.1 The American College of Medical Genetics issued a policy statement titled "Fragile X Syndrome: Diagnosis and Carrier Testing" in 1994 (Am J Med Genet 53:380-381). This document is also available online at www.faseb.org/genetics/acmg/ index.html. These Standards and Guidelines are in general agreement with that statement.

5.2 In 1995 the American College of Obstetrics and Gynecology issued a Committee Opinion, No. 161, on fragile X syndrome. These Standards and Guidelines are in general agreement with that opinion, with the exception of its recommendation for the use of amniocentesis for prenatal diagnosis. In experienced hands, chorionic villus samples are equally reliable and offer the advantage of first-trimester diagnosis to women who may be at $50 \%$ risk for an affected pregnancy. However, it cannot be stressed enough that the unique properties of this tissue must be recognized.

Approved by the Board of Directors of the American College of Medical Genetics on October 2, 2000.

- AMERICAN COLLEGE OF MEDICAL GENETICS, 2001

\section{Acknowledgments}

The authors offer sincere thanks to Drs. David Nelson, Ben Oostra, James MacPherson, Gert Matthijs, Jean-Louis Mandel, Ursula Froster, and Giovanni Neri for helpful early discussions, and Drs. Jack Tarleton, Nancy Carpenter, W. Ted Brown, and Sarah Nolin for their review of the final manuscript.

\section{References}

1. Rousseau F, Heitz D, Biancalana V, Blumenfeld S, Kretz C, Boue J, Tommerup N, Van Der Hagen C, DeLozier-Blanchet C, Croquette MF, et al. Direct diagnosis by DNA analysis of the fragile X syndrome of mental retardation. N Engl J Med 1991; 325:1673-1681.

2. Verkerk AJ, Pieretti M, Sutcliffe JS, Fu YH, Kuhl DP, Pizzuti A, Reiner O, Richards $\mathrm{S}$, Victoria MF, Zhang FP, et al. Identification of a gene (FMR-1) containing a CGG repeat coincident with a breakpoint cluster region exhibiting length variation in fragile X syndrome. Cell 1991;65:905-914.

3. Nakahori Y, Knight S, Holland K, Schwartz C, Roche A, Tarleton K, Wong S, Flint T, Froster-Iskenius U, Bentley D, Davies K, Hirst M. Molecular heterogeneity of the fragile X syndrome. Nucleic Acids Res 1991;19:4355-4359. 
4. Yu S, Pritchard M, Kremer E, Lynch M, Nancarrow J, Baker E, Holman K, Mulley JC Warren ST, Schlessinger D, Sutherland GR, Richards RI. Fragile X genotype characterized by an unstable region of DNA. Science 1991;252:1179-1182.

5. Fu Y-H, Kuhl DPA, Pizzuti A, Pieretti M, Sutcliffe JS, Richards S, Verkerk AJMH, Holden JJ, Fenwick RG, Warren ST, et al. Variation of the CGG repeat at the fragile X site results in genetic instability: resolution of the Sherman paradox. Cell 1991;67:1047-1058.

6. Yu S, Mulley J, Loesch D, Turner G, Donnelly A, Gedeon A, Hillen D, Kremer E, Lynch M, Pritchard M, Sutherland CR, Richards RI. Fragile-X syndrome: unique genetics of the heritable element. Am J Hum Genet 1992;50:968-980.

7. Erster SH, Brown WT, Goonewardena P, Dobkin CS, Jenkins EC, Pergolizzi RG. Polymerase chain reaction analysis of fragile X mutations. Hum Genet 1992;90:55-61.

8. Brown WT, Houck GE, Jeziorowska A, Levinson FN, Ding X, Dobkin C, Xhong N, Henderson J, Brooks SS, Jenkins EC. Rapid fragile X carrier screening and prenatal diagnosis using a non-radioactive PCR test. JAMA 1993;270:1569-1575.
9. Das S, Kubota T, Song M, Daniel R, Berry-Kravis EM, Prior TW, Popovich B, Rosser L, Arinami T, Ledbetter DH. Methylation analysis of the fragile X syndrome by PCR. Genet Test 1997-98;1:151-155.

10. Panagopoulos I, Lassen C, Kristoffersson U, Aman P. A methylation PCR approach for detection of fragile X syndrome. Hum Mutat 1999;14:71-79.

11. de Graaf E, Rouillard P, Willems P, Smits A, Rousseau F, Oostra B. Hotspot for deletions in the CGG repeat region of FMR1 in fragile X patients. Am J Med Genet 1996;64:301-308.

12. Marozzi A, Vegetti W, Manfredini E, Tibiletti MG, Testa G, Crosignani PG, Ginelli E, Meneveri R, Dalpra L. Association between idiopathic premature ovarian failure and fragile X premutation. Hum Reprod 1900;15:197-202.

13. Nolin SL, Lewis FA, Ye LL, Houck GE, Glicksman AE, Limprasert P, Li SY, Zhong N, Ashley AE, Feingold E, Sherman SL, Brown WT. Familial transmission of the FMR1 CGG repeat. Am J Hum Genet 1996;59:1252-1261.

This guideline is designed primarily as an educational resource for medical geneticists and other health care providers to help them provide quality medical genetic services. Adherence to this guideline does not necessarily ensure a successful medical outcome. This guideline should not be considered inclusive of all proper procedures and tests or exclusive of other procedures and tests that are reasonably directed to obtaining the same results. In determining the propriety of any specific procedure or test, the geneticist should apply his or her own professional judgment to the specific clinical circumstances presented by the individual patient or specimen. It may be prudent, however, to document in the patient's record the rationale for any significant deviation from this guideline. 